\title{
Partite Turán-densities for complete runiform hypergraphs on $r+1$ vertices
}

\author{
Markström, Klas; Thomassen, Carsten
}

Published in:

The Electronic Journal of Combinatorics

Link to article, DOI:

10.4310/JOC.2021.v12.n2.a3

Publication date:

2021

Document Version

Peer reviewed version

Link back to DTU Orbit

Citation (APA):

Markström, K., \& Thomassen, C. (2021). Partite Turán-densities for complete nuniform hypergraphs on $r+1$ vertices. The Electronic Journal of Combinatorics, 12(2), 235-245. https://doi.org/10.4310/JOC.2021.v12.n2.a3

\section{General rights}

Copyright and moral rights for the publications made accessible in the public portal are retained by the authors and/or other copyright owners and it is a condition of accessing publications that users recognise and abide by the legal requirements associated with these rights.

- Users may download and print one copy of any publication from the public portal for the purpose of private study or research.

- You may not further distribute the material or use it for any profit-making activity or commercial gain

- You may freely distribute the URL identifying the publication in the public portal

If you believe that this document breaches copyright please contact us providing details, and we will remove access to the work immediately and investigate your claim 


\title{
Partite Turán-densities for complete $r$-uniform hypergraphs on $r+1$ vertices
}

\author{
Klas Markström*and Carsten Thomassen ${ }^{\dagger}$
}

May 7, 2020

\begin{abstract}
In this paper we investigate density conditions for finding a complete $r$-uniform hypergraph $K_{r+1}^{(r)}$ on $r+1$ vertices in an $(r+1)$-partite $r$ uniform hypergraph $G$. First we prove an optimal condition in terms of the densities of the $(r+1)$ induced $r$-partite subgraphs of $G$. Second, we prove a version of this result where we assume that $r$-tuples of vertices in $G$ have their neighbours evenly distributed in $G$. Third, we also prove a counting result for the minimum number of copies of $K_{r+1}^{(r)}$ when $G$ satisfies our density bound, and present some open problems.

A striking difference between the graph, $r=2$, and the hypergraph, $r \geq 3$, cases is that in the first case both the existence threshold and the counting function are non-linear in the involved densities, whereas for hypergraphs they are given by a linear function. Also, the smallest density of the $r$-partite parts needed to ensure the existence of a complete $r$-graph with $(r+1)$ vertices is equal to the golden ratio $\tau=0.618 \ldots$ for $r=2$, while it is $\frac{r}{r+1}$ for $r \geq 3$.
\end{abstract}

\section{Introduction}

One of the classical problems in extremal graph theory is that of finding the maximum density of a graph $G$ which does not contain some fixed graph $H$. This density is known as the Turán density for $H$ and is defined as $\pi(H)=$ $\lim _{n \rightarrow \infty} \frac{e x(n, H)}{\left(\begin{array}{c}n \\ 2\end{array}\right)}$, where the Turán number $e x(n, H)$ is the maximum number of edges in a graph on $n$ vertices which does not have $H$ as a subgraph. Mantel proved that $\pi\left(K_{3}\right)=\frac{1}{2}$ and later Turán gave a complete answer for $H=K_{t}$ [Tur41].

Motivated by a question of Erdős regarding the maximum density of a triangle free subgraph of a graph [BSTT06] investigated a modified version of the Turán density, namely that of finding the maximum density of a 3-partite graph which does not contain a $K_{3}$. The problem was given a complete solution in terms of the three bipartite graphs induced by each pair of vertex classes of the 3-partition. Later [BJT10] investigated the number of $K_{3} \mathrm{~s}$ as a function of

\footnotetext{
${ }^{*}$ Department of Mathematics and Mathematical Statistics, Umeå University, SE-901 87 Umeå, Sweden

${ }^{\dagger}$ Department of Applied Mathematics and Computer Science, Technical University of Denmark, DK-2800 Lyngby, Denmark
} 
these densities, and a sharp result was given for large enough densities. Enumeration of triangles in general graphs has a long history and was finally solved by Razborov [Raz08].

Our aim in this paper is to investigate generalisations of these questions to uniform hypergraphs. In particular we will determine the maximal density of an $(r+1)$-partite $r$-uniform hypergraph which does not contain the complete hypergraph $K_{r+1}^{r}$. We will also present a sharp bound on the number of copies of $K_{r+1}^{r}$. These results demonstrate a qualitative difference between graphs and hypergraphs with $r \geq 3$, where interestingly enough the extension to the hypergraph case is less complex than the graph case. The existence condition found in [BSTT06] for graphs is non-linear in terms of the involved densities, as are the counting results from [BJT10], but as our results show the corresponding condition, and counting function, for $r \geq 3$ are given by simple linear functions.

For hypergraphs far less is known in the non-partite case than for graphs. Turán conjectured that $\pi\left(K_{4}^{3}\right)=\frac{5}{9}$, and gave a matching construction for the lower bound. Using flag-algebra Razborov [Raz10] has proven that $\pi\left(K_{4}^{3}\right) \leq$ 0.56167. For $r=4$ Giraud [Gir90] gave a construction which shows that $\pi\left(K_{5}^{4}\right) \geq \frac{11}{16}$, and Sidorenko [Sid95] conjectured that this is in fact the correct value. The best current upper bound $\pi\left(K_{5}^{4}\right) \leq \frac{1753}{2380}$ was given by the first author in [Mar09]. For $r \geq 5$ much less is known. De Caen [dC83] proved that $\pi\left(K_{r+1}^{r}\right) \leq\left(1-\frac{1}{r}\right)$, and this was later sharpened somewhat for odd $r$ in [CL99] and even $r$ in [LZ09]. As a corollary to one of our results we will get a short proof of de Caen's bound.

Mubayi and Talbot [MT08] investigated the global density of a 4-partite 3-graph $G$ such that $K_{4}^{3} \not \subset G$. and proved the sharp result $|E(G)| \leq \frac{8}{17}\left(\begin{array}{c}|V(G)| \\ 3\end{array}\right)$.

One of the few counting results for hypergraphs is by Mubayi [Mub13] who used the hypergraph removal lemma [Gow07, NRS06, RS06, Tao06] to prove lower bounds on the number of copies of $H$ in a hypergraph with ex $(n, H)+$ $q$ edges. These bounds apply to certain forbidden hypergraphs $H$ with the property that there is a unique $H$-free hypergraph on $\operatorname{ex}(n, H)$ edges, and the bounds are of the form $q c(n, H)$, where $c(n, H)$ is the minimum number of copies of $H$ in any $n$-vertex $r$-graph on $\operatorname{ex}(n, H)+1$ edges.

\subsection{Definitions}

For $r \geq 2$ we will refer to an $r$-uniform hypergraph as an $r$-graph. An $r$-graph $G$ is $t$-partite if its vertex has a partition in vertex classes $V_{1}, \ldots, V_{t}$, such that each edge has at most one vertex in any class $V_{i}$.

Definition 1.1. Given a $t$-partite $r$-graph $G$ with vertex classes $V_{1}, \ldots, V_{t}$ and a set of indices $I$ we let $P_{I}$ denote the $(t-|I|)$-partite $r$-graph induced by the classes with indices not in $I$. If $I=\{i\}$ then we abbreviate this as $P_{i}$

As in [BSTT06] and [BJT10] we are going to work with vertex weighted $r$-graphs. We assume that $G$ has a weight-function $w$ defined on its vertices. The weight of a set $S$ of vertices is $w(S)=\sum_{v \in S} w(v)$.

We define the weight of an edge $e=\left\{v_{1}, \ldots, v_{r}\right\}$ to be the product $\prod_{i=1}^{r} w\left(v_{i}\right)$, and the weight of a set of edges as the sum of their weights.

An unweighted $r$-graph will here be seen as the same graph with a weight function $w(v)=1$ for all vertices $v$. 
Definition 1.2. Given an $(r+1)$-partite $r$-graph $G$ we let $\rho(i)$ be the density of the $r$-partite graph induced by $P_{i}$, which is $\frac{w\left(E\left(P_{i}\right)\right)}{\prod_{j \neq i} w\left(V_{j}\right)}$, and $\bar{\rho}$ is the vector of these densities.

\section{Threshold and the minimum number of $K_{r+1}^{r}$ in an $(r+1)$-partite $r$-graph}

Given an $(r+1)$-partite $r$-graph we want to count the number of $K_{r+1}^{r} \mathrm{~s}$ it contains. It will be convenient to do this in terms of the density of $K_{r+1}^{r} \mathrm{~s}$

Definition 2.1. Given an $(r+1)$-partite $r$-graph $G$ we define the density of $K_{r+1}^{r}$ s in $G$ to be

$$
C(G)=\left(\sum_{S=K_{r+1}^{r} \subset G} \prod_{v \in S} w(s)\right) / \prod_{i=1}^{r+1} w\left(V_{i}\right)
$$

We define the minimum density of $K_{r+1}^{r} \mathrm{~s}$ as

$$
C(\bar{\rho})=\inf _{G} C(G),
$$

where the infimum is taken over all $(r+1)$-partite $r$-graphs with density $\bar{\rho}$.

We will need the following polynomial from [BJT10].

Definition 2.2. $\Delta(a, b, c)=a^{2}+b^{2}+c^{2}-2 a b-2 a c-2 b c+4 a b c$

The proof of the following lemma is a routine, but somewhat lengthy, calculus exercise.

Lemma 2.3. If $a, b, c$ are real numbers in the interval $[0,1]$ and $a+b+c \geq \frac{9}{4}$ then $\Delta(a, b, c) \geq 0$, and $a b+c>1, a c+b>1$, and $b c+a>1$.

In particular if each if each of $a, b$ and $c$ is at least $\frac{3}{4}$ then $\Delta(a, b, c) \geq 0$.

The following theorem was proven in [BJT10].

Theorem 2.4. The minimum density of triangles in a tripartite graph with edge densities given by $(a, b, c)$ such that $\Delta(a, b, c) \geq 0, a b+c>1, a c+b>1$, and $b c+a>1$, is given by $a+b+c-2$. of $r$.

We will fist extend the lower bound given by this theorem to larger values

Lemma 2.5. If $\bar{\rho}$ is a vector with rational numbers between 0 and 1 , such that

$$
\sum_{i=1}^{r+1} \rho(i)-r \geq 0
$$

then there exists a weighted $(r+1)$-partite $r$-graph $G$ with rational weights such that $\bar{\rho}$ is the density vector of $G$ and $C(G)=\sum_{i=1}^{r+1} \rho(i)-r$. 
Proof. We will prove the statement by induction on $r$. For $r=2$ the statement is true by Theorem 2.4 of [BJT10], as stated earlier. By that theorem there is an optimal graph which is a weighted 3-partite graph on six vertices whose 3 -partite complement is a perfect matching. Hence we only need to prove the statement for $r \geq 3$.

Without loss of generality we assume that $\rho(i) \geq \rho(i+1)$ for $1 \leq i \leq r$ and hence, by averaging, that $\rho(1)+\rho(2)+\rho(3) \geq \frac{3 r}{r+1} \geq \frac{9}{4}$. Thus, by Lemma 2.3, we know that the conditions of Theorem 2.4 are satisfied by these three values.

We now assume that the statement is true for $r-1$. In order to build an $r$-graph $H_{2}$ which satisfies the statement in the Lemma we take an $r$-partite $(r-1)$-graph $H_{1}$, with the first $r$ parts of $\bar{\rho}$ as its density vector, which satisfies the statement for $r-1$, and build an $(r+1)$-partite $r$-graph $H_{2}$ by adding one new class $V_{r+1}$ which contains a single vertex $v$, with weight 1 .

For every edge $e$ in $H_{1}$ we let $e \cup\{v\}$ be an edge of $H_{2}$. This means that for $i \neq r+1$ the density contributed to $\rho(i)$ by these edges will be the same as in $H_{1}$. Hence $H_{2}$ has the desired density $\rho(i)$ for these classes, and the edges added so far do not give rise to a $K_{r+1}^{r}$.

We will now add edges among the first $r$ classes. One of these edges will be part of a weighted $K_{r+1}$ in $H_{2}$ if the corresponding $r$-tuple in $H_{1}$ is a $K_{r}^{(r-1)}$ in $H_{1}$, and by induction we know the density of such $K_{r}^{(r-1)}$.

As pointed out in [BJT10] we may assume that $H_{1}$ is in fact a simple unweighted hypergraph, since a hypergraph with rational weights can be modified by a suitable blow-up into an equivalent unweighted hypergraph on a larger number of vertices.

In $H_{1}$ the density of partite $r$-tuples which do not span a $K_{r}^{(r-1)}$ is $1-$ $\left(\sum_{i=1}^{r} \rho(i)-(r-1)\right)$, and all these tuples can be added as edges without creating a $K_{r+1}^{r}$ in $H_{2}$. Each additional edge added after these form a unique $K_{r+1^{r}}$ together with the vertex in $V_{r+1}$, so if we add enough edges to reach the desired density $\rho(r+1)$ we will have

$$
\rho(r+1)-\left(1-\left(\sum_{i=1}^{r} \rho(i)-(r-1)\right)\right)=\sum_{i=1}^{r+1} \rho(i)-r
$$

density of $K_{r+1}^{r}$ in $H_{2}$.

Our next step will to prove a lower bound for the density of $K_{r} \mathrm{~s}$.

Theorem 2.6. Let $G$ be an $(r+1)$-partite $r$-graph, then the density of $K_{r+1}^{r} s$ satisfies the following inequalities

1. $C(\bar{\rho}) \geq\left(\sum_{i=1}^{r+1} \rho(i)\right)-r$.

2. If $\bar{\rho}$ satisfies the conditions of Lemma 2.5 then $C(\bar{\rho})=\left(\sum_{i=1}^{r+1} \rho(i)\right)-r$.

Proof. 1. Let $\mathbf{1}(e)$, where $e$ is a set of size $r$, be 1 if $e$ is an edge of $G$ and 0 otherwise, let $\mathbf{1}(H)$ be 1 if $H$ is the vertex set of a $K_{r+1}$ contained in $G$, and 0 otherwise.

Now

$$
\sum_{H} w(H)\left(\sum_{i \in H} \mathbf{1}(H \backslash i)\right) \leq \sum_{H} w(H)(r+\mathbf{1}(H))
$$


But the left hand side is the sum of the densities in $G$ and the right hand side is $r$ plus the density of $K_{r+1}^{r}$ s so

$$
\sum_{i} \rho(i) \leq r+C(\bar{\rho})
$$

and so $C(\bar{\rho}) \geq \sum_{i} \rho(i)-r$, and we have proven part 1 .

2. Lemma 2.5 shows that the bound in Theorem 2.6 is sharp, and that for rational densities satisfying the inequality in Lemma 2.4 equality is achieved for some finite $r$-graph.

Corollary 2.7. If all densities $\rho(i)>\frac{r}{r+1}$ in $G$ then $K_{r+1}^{r} \subset G$

If $r \geq 3$ and $\rho(i)=\rho(j)$, for all $i, j$ and $\rho(i) \leq \frac{r}{r+1}$ then there exists $G$ with these densities such that $K_{r+1}^{r} \not \subset G$

We note that the graph which achieves the minimum number of copies of $K_{r+1}^{r} \mathrm{~s}$ is not unique, i.e.the $(r+1)$-partite Turán-problem for $K_{r+1}^{r}$ is not stable. Byt the existing results for $r=2$ we know that there is not a unique graph which minimizes the number of triangles and Lemma 2.5 gives distinct extensions to higher values of $r$.

\section{Balanced Codegrees}

Our second result concerns degrees rather than densities, and for $r$-graph we have found it natural to consider the degrees of the $(r-1)$-tuples of vertices in $G$.

Definition 3.1. Given a multipartite $r$-graph $G$, with $V(G)$ partitioned into at least $r$ classes, we say that a $t$-tuple $g$ of vertices from $G$ is partite if it has at most one vertex in each class of $G$.

Definition 3.2. Given a partite $t$-tuple $g$ we say that an $(r-t)$-tuple $h$ is completing $g$ if $(g \cup h) \in E(G)$. We call the set of completing $(r-t)$-tuples for $g$ the neighbourhood $N(g)$ of $g$. The neighbourhood of $g$ in a set $I$ of classes is the set of completing $(r-t)$-tuples in $I$, and is denoted $N(I, g)$

The degree of $g$ is $d(g)=|N(g)|$ and the degree in an $(r-t)$-tuple $I$ of classes is $d(I, g)=|N(I, g)|$.

By the minimum codegree of an (multipartite) $r$-graph $G$ we refer to the minimum degree of all (partite) $(r-1)$-tuples of vertices in $V(G)$

Definition 3.3. We say that a partite $t$-tuple $g$ of vertices in $G$ has strictly balanced degree if it has the same number of completing $(r-t)$-tuples in each of the $(r-t)$-tuples of classes which $g$ does not intersect.

Theorem 3.4. If the partite $(r-1)$-tuples of $G$ have strictly balanced degrees and $\max _{j} \sum_{i \neq j} \rho(i)>(r-1)$ then $K_{r+1}^{r} \subset G$.

Note that the condition on the partite $(r-1)$-tuples means that such a tuple splits its neighbourhood equally between each of the two parts which the tuple does not intersect, but the sizes of those neighbourhoods may differ between different tuples. 
Proof. We will first look at the case $j=1$. Let $e$ be an edge of $P_{1}$ and let $g_{2}, \ldots, g_{r+1}$ be the $(r-1)$-tuples which are subsets of $e$. If $G$ does not contain $K_{r+1}^{r}$ we must have that

$$
S(e)=\sum_{i=2}^{r+1} d\left(V_{1}, g_{i}\right) \leq(r-1)
$$

since otherwise there would be a common vertex in the neighbourhoods of the $(r-1)$-tuples, and we would have a $K_{r+1}^{r}$.

If we sum over all edges in $P_{1}$ we find that

$$
\sum_{e \in E\left(P_{1}\right)} S(e) \leq(r-1) \rho(1) \prod_{k \neq 1}\left|V_{k}\right|
$$

or equivalently

$$
\sum_{i=2}^{r+1} \sum_{g \in P_{\{1, i\}}} d\left(V_{1}, g\right) d\left(V_{i}, g\right) \leq(r-1) \rho(1) \prod_{k \neq 1}\left|V_{k}\right|
$$

where the inner sum is over all partite $(r-1)$-tuples $g$.

Using the fact that each $g$ has strictly balanced degree we rewrite this as

$$
\sum_{i=2}^{r+1} \sum_{g \in P_{\{1, i\}}} d\left(V_{1}, g\right)^{2} \leq(r-1) \rho(1) \prod_{k \neq 1}\left|V_{k}\right|
$$

Using the Cauchy-Schwarz inequality we get

$$
\sum_{i=2}^{r+1} \frac{\left(\sum_{g \in P_{\{1, i\}}} d\left(V_{1}, g\right)\right)^{2}}{\prod_{k \neq 1, i}\left|V_{k}\right|} \leq(r-1) \rho(1) \prod_{k \neq 1}\left|V_{k}\right|
$$

By definition

$$
\sum_{g \in P_{\{1, i\}}} d\left(V_{1}, g\right)=\rho(i) \prod_{k \neq i}\left|V_{k}\right| .
$$

Substituting this we find that

$$
\sum_{i=2}^{r+1} \rho(i)^{2} \prod_{k \neq i}\left|V_{k}\right| \leq(r-1) \rho(1) \prod_{k \neq 1}\left|V_{k}\right|
$$
to get

But $\rho(i) \prod_{k \neq i}\left|V_{k}\right|$ is independent of $i$ so we can divide both sides by $\rho(1) \prod_{k \neq 1}\left|V_{k}\right|$

$$
\sum_{i=2}^{r+1} \rho(i) \leq(r-1)
$$

The results follows for other values of $j$ in the same way.

As shown in [BSTT06] this result is sharp for $r=2$, but we do not have a matching lower bound for larger $r$.

Relating to the case where all densities are equal we get the following 
Corollary 3.5. If all partite $(r-1)$-tuples of $G$ have strictly balanced degree and $\rho(i)>1-\frac{1}{r}$ for all $i$ then $K_{r+1}^{r} \subset G$.

This can be compared to the minimum codegree which forces a $K_{r+1}^{r}$ in the non-partite case. In [LM14] it was proven that there are $r$-graph with minimum $(r-1)$-degree $\frac{1}{2}(n-2)$ which do not contain a $K_{r+1}^{r}$, and it was conjectured that this is an optimal bound. This in turn implies that the global density is at least $\frac{1}{2}$.

Another simple corollary of this result is de Caen's upper bound on the Turán-density of $K_{r+1}^{r}$.

Corollary 3.6 (de Caen [dC83]). If $G$ is an r-graph and $|E(G)|>\left(1-\frac{1}{r}\right) \frac{n^{r}}{r !}$ then $K_{r+1}^{r} \subset G$.

Proof. Given a labelled $r$-graph $G$ with vertex set $V$ we will form a new $(r+1)$ partite $r$-graph $H$. The vertex set of $H$ is the disjoint union of $r+1$ copies $V_{1}, \ldots, V_{r+1}$ of $V$.

Given an edge $e=\left\{v_{1}, v_{2}, \ldots, v_{r}\right\}$ of $G$, a choice of $r$ of the classes $V_{i}$, and a permutation $\pi \in S_{r}$, we let $e_{\pi}=\left\{v_{\pi(1)}, v_{\pi(2)}, \ldots, v_{\pi(r)}\right)$, where the $j$ th vertex in the tuple is the vertex in the $j$ th of the $r$ vertex classes, be an edge in the $r$-partite subgraph of $H$ induced by those classes. Note that in this way each edge $e$ of $G$ gives rise to $r$ ! edges in each of the $r$-partite subgraphs of $H$. (So if we look at ordinary graphs and let $G$ be a single edge $(1,2)$ then $H$ would become a 6 -cycle which winds twice through the three parts.)

The $r$-graph $H$ has strictly balanced codegrees, since the number of neighbours in $V_{i}$, of a partite $(r-1)$-tuple $t$ in $H$, only depends on whether $t$ intersects $V_{i}$ or not, and the number of neighbours of $t$ in $G$.

By the assumptions each $\rho(i)$ in $H$ is strictly greater than $\left(1-\frac{1}{r}\right)$, so $H$ contains a copy of $K_{r+1}^{r}$, and the corresponding vertices form a $K_{r+1}^{r}$ in $G$.

In fact the proof of Theorem 3.4 can easily be modified to give a bound for the degree required to give a copy of $\left(K_{r+1}^{r}-k\right)$, the $r$-graph obtained by deleting any $k$ edges from $K_{r+1}^{r}$, we just need to modify the first bound for $S(e)$ to be less than $r-k-1$. This gives

Theorem 3.7. If the partite $(r-1)$-tuples of $G$ have strictly balanced degrees, $k \leq r-1$, and $\max _{j} \sum_{i \neq j} \rho(i)>r-k-1$ then $\left(K_{r+1}^{r}-k\right) \subset G$.

For $k \geq r-1$ the graph $\left(K_{r+1}^{r}-k\right)$ is in fact $r$-partite, and the strictly balanced degree condition means that if $G$ has at least one edge then any edge will be part of a $\left(K_{r+1}^{r}-(r-1)\right)$, which is simply two edges overlapping on an $(r-1)$-tuple.

Using this theorem as in the proofs of Corollaries 3.5 and 3.6 we get

Corollary 3.8. If all partite ( $r-1)$-tuples of $G$ have balanced degree, $k \leq(r-1)$, and $\rho(i)>1-\frac{k+1}{r}$ for all $i$ then $\left(K_{r+1}^{r}-k\right) \subset G$.

Corollary 3.9. If $G$ is an $r$-graph, $k \leq(r-1)$, and $|E(G)|>\left(1-\frac{k+1}{r}\right) \frac{n^{r}}{r !}$ then $\left(K_{r+1}^{r}-k\right) \subset G$.

The case $r=3$ of the latter result was proven by de Caen in [dC83]. 


\section{Open problems}

Following [BSTT06] and the results in this paper concerning the codegree it is natural to ask what happens for general degrees in $r$-graphs with $r>2$. The first open case is vertex degrees for 3-graphs

Question 4.1. Let $G$ be a 4-partite 3-graph with balanced vertex degrees. Which densities force a $K_{4}^{3}$ in $G$ ?

For $r>3$ the corresponding question is open for balanced $l$-degrees for all $l<r-1$.

Question 4.2. Let $G$ be an $r$-partite $r$-graph with $4 \leq r$. Which densities forces a $K_{r+1}$ if the partite l-tuples have balanced degrees, where $1 \leq l<r-1$.

Let $G$ be a $t$-partite $r$-graph with all densities at least $\alpha$. Given an $r$-graph $H$, we can ask for the minimum density $\alpha$ which forces a copy of $H$ in $G$. We call this density $d_{t}(H)$.

Question 4.3. Does there exist a finite $t_{0}$ such that $d_{t}(H)=d_{t_{0}}(H)$ for $t \geq t_{0}$ ?

For $r=2$ and $H=K_{t}$ the answer is yes by [Pfe12].

\section{Acknowledgement}

This research was done while the authors were attending the research semester Graphs, hypergraphs and computing at Institut Mittag-Leffler (Djursholm, Sweden). The first author was supported by The Swedish Research Council grant 2014-4897. The second author was supported by ERC Advanced Grant GRACOL.

\section{References}

[BJT10] Rahil Baber, J. Robert Johnson, and John Talbot, The minimal density of triangles in tripartite graphs, LMS J. Comput. Math. 13 (2010), 388-413. MR 2685132 (2011g:05254)

[BSTT06] Adrian Bondy, Jian Shen, Stéphan Thomassé, and Carsten Thomassen, Density conditions for triangles in multipartite graphs, Combinatorica 26 (2006), no. 2, 121-131. MR 2223630 (2007a:05062)

[CL99] Fan Chung and Linyuan Lu, An upper bound for the Turán number $t_{3}(n, 4)$, J. Combin. Theory Ser. A 87 (1999), no. 2, 381-389. MR 1704268 (2000d:05060)

[dC83] Dominique de Caen, Extension of a theorem of Moon and Moser on complete subgraphs, Ars Combin. 16 (1983), 5-10. MR 734038 (85d:05132)

[Gir90] Guy R. Giraud, Remarques sur deux problèmes extrémaux, Discrete Math. 84 (1990), no. 3, 319-321. MR 1077144 (91g:05073) 
[Gow07] W. Timothy Gowers, Hypergraph regularity and the multidimensional Szemerédi theorem, Ann. of Math. (2) 166 (2007), no. 3, 897-946. MR 2373376 (2009d:05250)

[LM14] Allan Lo and Klas Markström, $\ell$-degree turan density, SIAM Journal on Discrete Mathematics 28 (2014), no. 3, 1214-1225.

[LZ09] Linyuan Lu and Yi Zhao, An exact result for hypergraphs and upper bounds for the Turán density of $K_{r+1}^{r}$, SIAM J. Discrete Math. 23 (2009), no. 3, 1324-1334. MR 2538655 (2011e:05268)

[Mar09] Klas Markström, Extremal hypergraphs and bounds for the Turán density of the 4-uniform $K_{5}$, Discrete Math. 309 (2009), no. 16, 5231-5234. MR 2548922 (2010i:05236)

[MT08] Dhruv Mubayi and John Talbot, Extremal problems for t-partite and t-colorable hypergraphs, Electron. J. Combin. 15 (2008), no. 1, Research Paper 26, 9. MR 2383446 (2009e:05304)

[Mub13] Dhruv Mubayi, Counting substructures ii: Hypergraphs, Combinatorica 33 (2013), no. 5, 591-612.

[NRS06] Brendan Nagle, Vojtěch Rödl, and Mathias Schacht, The counting lemma for regular $k$-uniform hypergraphs, Random Structures Algorithms 28 (2006), no. 2, 113-179. MR 2198495 (2007d:05084)

[Pfe12] Florian Pfender, Complete subgraphs in multipartite graphs, Combinatorica 32 (2012), no. 4, 483-495. MR 2965288

[Raz08] Alexander A. Razborov, On the minimal density of triangles in graphs, Combin. Probab. Comput. 17 (2008), no. 4, 603-618. MR 2433944 (2009i:05118)

[Raz10] , On 3-hypergraphs with forbidden 4-vertex configurations, SIAM J. Discrete Math. 24 (2010), no. 3, 946-963. MR 2680226 (2011k:05171)

[RS06] Vojtěch Rödl and Jozef Skokan, Applications of the regularity lemma for uniform hypergraphs, Random Structures Algorithms 28 (2006), no. 2, 180-194. MR 2198496 (2006j:05099)

[Sid95] Alexander Sidorenko, What we know and what we do not know about Turán numbers, Graphs Combin. 11 (1995), no. 2, 179-199. MR 1341481 (96f:05053)

[Tao06] Terence Tao, A variant of the hypergraph removal lemma, J. Combin. Theory Ser. A 113 (2006), no. 7, 1257-1280. MR 2259060 (2007k:05098)

[Tur41] Paul Turán, Eine Extremalaufgabe aus der Graphentheorie, Mat. Fiz. Lapok 48 (1941), 436-452. MR 0018405 (8,284j) 